\title{
Is Implicit Motor Imagery a Reliable Strategy for a Brain-Computer Interface?
}

\author{
Bethel A. Osuagwu, Magdalena Zych, and Aleksandra Vuckovic
}

\begin{abstract}
Explicit motor imagery (eMI) is a widely used brain-computer interface $(\mathrm{BCl})$ paradigm, but not everybody can accomplish this task. Here, we propose a $\mathrm{BCl}$ based on implicit motor imagery (iMI). We compared classification accuracy between $\mathrm{eMI}$ and $\mathrm{iMI}$ of hands. Fifteen able-bodied people were asked to judge the laterality of hand images presented on a computer screen in a lateral or medial orientation. This judgment task is known to require mental rotation of a person's own hands, which in turn is thought to involve iMI. The subjects were also asked to perform eMI of the hands. Their electroencephalography was recorded. Linear classifiers were designed based on common spatial patterns. For discrimination between left hand and right hand, the classifier achieved maximum of $81 \pm 8 \%$ accuracy for eMI and $83 \pm 3 \%$ for iMI. These results show that iMI can be used to achieve similar classification accuracy as eMI. Additional classification was performed between iMI in medial and lateral orientations of a single hand; the classifier achieved $81 \pm 7 \%$ for the left hand and $78 \pm 7 \%$ for the right hand, which indicate distinctive spatial patterns of cortical activity for iMI of a single hand in different directions. These results suggest that a special $\mathrm{BCl}$ based on iMI may be constructed, for people who cannot perform explicit imagination, for rehabilitation of movement, or for treatment of bodily spatial neglect.
\end{abstract}

Index Terms-EEG, mental rotation, motor imagery, sensorimotor cortex activation, $\mathrm{BCl}$.

\section{INTRODUCTION}

$\mathbf{E}$ XPLICIT motor imagery (eMI) of left and right hand is a widely used paradigm for brain computer interfaces (BCI) applications in neurorehabilitation [1], [2]. In this paradigm, participants are explicitly asked to imagine to move their hands guided by a visual cue presented on a computer screen. Distinctive spatial patterns of activation during eMI of different limbs enable machine learning algorithms to

Manuscript received December 12, 2016; revised April 25, 2017; accepted May 29, 2017. Date of publication June 29, 2017; date of current version November 29, 2017. This work was supported by EPSRC through a Ph.D. Scholarship under Grant EP/P505534/1. (Corresponding author: Bethel A. Osuagwu.)

B. A. Osuagwu was with the Centre for Rehabilitation Engineering, University of Glasgow, G12 8QQ Glasgow, U.K. He is now with Stoke Mandeville Spinal Research, National Spinal Injuries Centre, Stoke Mandeville Hospital, Buckinghamshire NHS Trust, HP21 8AL Aylesbury, U.K. (e-mail: bethel.osuagwu@gmail.com).

M. Zych was with the Centre for Rehabilitation Engineering, University of Glasgow, G12 8QQ Glasgow, U.K. She is now with the School of Electrical and Electronic Engineering, University College Dublin, D04 V1W8 Dublin, Ireland.

A. Vuckovic is with the Centre for Rehabilitation Engineering, University of Glasgow, G12 8QQ Glasgow, U.K.

Digital Object Identifier 10.1109/TNSRE.2017.2712707 successfully classify these two types of eMI. Although eMI is widely used, a study showed that approximately $93 \%$ of healthy people tested could achieve an accuracy of $60 \%$ using an eMI-based BCI [3]. Another study showed that $11 \%$ of patients with stroke could not operate an eMI-based BCI [4].

There is evidence showing that mental rotation of a body part involves motor imagery of that body part [5]-[7]. This is because mental rotation of a body part activates similar areas of the motor cortex as eMI [5]-[7]. Mental rotation can be induced during laterality judgement. For example, hand mental rotation is induced during hand laterality test (HLT). In HLT a subject is asked to judge the laterality of individual hand pictures presented with different gestures and rotations. In order to achieve this judgement without physically moving the hands, a subject has to mentally rotate his/her own hand to align it with the presented hand picture [8]. Motor imagery of hand and mental rotation of hand in HLT share a common characteristic because they both activate the sensorimotor areas of the hand [5]. Given that mental rotation of hand involves mental movement of one's own hand, it is more likely to be similar to first-person than third-person eMI. The activation of the sensorimotor cortex by mental rotation process shows that the process does not involve only visual but also proprioceptive imagination of movement [9]. Unlike during eMI, subjects are not necessarily aware of movement imagination during mental rotation and for this reason the motor imagination involved in mental rotation is referred to as implicit motor imagery (iMI).

Implicit motor imagery induced during HLT has been used clinically in 'graded motor imagery' treatment of patients with complex regional pain syndrome, who have asymmetrical presentation of healthy and affected hand in primary sensory cortex [10]. In graded motor imagery protocol, iMI is used as a first step preceding eMI training in patients who feel pain and discomfort when performing eMI of their affected limbs [6].

In addition to pain treatment, iMI in mental rotation could also be used in rehabilitation of movement, as it activates not only cortical areas responsible for visuo-spatial integrations, but also the sensorimotor cortex, in a similar manner to eMI [5]. Further, it could be used in stroke patients with spatial neglect for implicitly involving the neglected side of the body.

Using iMI could have an advantage over eMI given that the former may not depend on the capacity of an individual to consciously imagine movements. The capacity to imagine movement may be measured by ability to control a motor imagery based BCI [3], [4], [11] or more conventionally by motor imagery questionnaires [11], [12]. Healthy people 
vary in their ability to perform kinaesthetic imagination of movement, mental rehearsal of kinaesthetic rather than visual properties of movement [12], and this ability is further reduced due to various neurological conditions such as stroke or spinal cord injury [13]. Implicit motor imagery is an automatic process that activates the sensorimotor cortex [7], [14], [15]. This is unlike in eMI where conscious/explicit imagination is needed to activate sensorimotor cortex.

In our previous study we compared EEG recording during cue based eMI and iMI during HLT [5]. We showed that similar lateralisation exist while performing both tasks, though cortical response in iMI was delayed due to time required to make a laterality judgement. Although we showed that the differences exist, we did not quantify the degree of discrimination that can be achieved when classifying between left and right hand iMI. If similar accuracies achieved using eMI can be achieved with iMI, a special BCI channelled for rehabilitation could be constructed. This BCI could be implemented in patients who are unable to perform eMI due to, for example, loss of proprioception/sensation following spinal cord injury or stroke. In addition, HLT or in general mental rotation which induces the iMI can be more entertaining as it involves observation of a variety of pictures showing hands with various gestures and can be seen as a computer game. A BCI based on iMI may have a delayed feedback, provided after each single trial. This type of feedback has been previously successfully used to train people to voluntarily modulate their H-reflex using delayed information about their muscle activity [16].

In this paper we use an offline classifier with the aim to answer the following questions: (i) is it possible to achieve comparable classification accuracies in both eMI and iMI? (ii) is it possible to classify iMI based only on the activity of the sensorimotor cortex? (iii) Is it possible to discriminate between the medial and lateral mental rotation of the same hand? Positive answers to these questions may indicate that iMI could be used to create a BCI for rehabilitation of movement or for treatment of spatial neglect in stroke patients.

\section{METHODS}

\section{A. Data Collection}

1) Subjects: Data from 15 right handed healthy subjects who gave their informed consents were used for this study. The subjects with mean age of $24.9 \pm 19$ included six females and nine males. The study was approved by the University ethics committee.

2) Explicit MI Trials: A software program, rtsBCI [17] was used to implement a cue-based paradigm. One trial took $6000 \mathrm{~ms}$. At the start of a trial $(\mathrm{t}=-3000 \mathrm{~ms})$, a subject was presented with a blank screen. A warning cue (a cross) was shown on the screen at $t=-1000$ ms notifying the subject to get ready. The cross vanished at the end of the trial which was at $\mathrm{t}=3000 \mathrm{~ms}$. From $\mathrm{t}=-3000 \mathrm{~ms}$ to $\mathrm{t}=0 \mathrm{~ms}$, the subjects were instructed to remain relaxed. This period is the rest period when they were not performing any study related task. At time $\mathrm{t}=0 \mathrm{~ms}$ an execution cue (an arrow pointing to the left or right) appeared on the screen and remained till $\mathrm{t}=1250 \mathrm{~ms}$. Depending on the current cue on the screen, the subjects had to practice continuous kinaesthetic eMI of opening and closing of the left or the right hand. This gave two types of eMI condition which are: right hand eMI and left hand eMI. The subjects were instructed to continuously imagine the hand movement from $\mathrm{t}=0 \mathrm{~ms}$ till $\mathrm{t}=3000 \mathrm{~ms}$. They were instructed to stop the imagination and rest from $\mathrm{t}=3000 \mathrm{~ms}$ for a variable length of time (ranging from 1000 to $3000 \mathrm{~ms}$ ) before another trial started. This often used brain computer interface paradigm [11] is presented in Fig. 1a.

3) Implicit MI Trials: Note that iMI trials refers to HLT trials. Following similar timing as in eMI trials, at $\mathrm{t}=-1000 \mathrm{~ms}$ the warning cue was presented. This warning cue was accompanied with a beep sound. The beep sound serves as reference for extracting the responses of the subjects on an audio recording explained later. At $\mathrm{t}=0 \mathrm{~ms}$, an execution cue was presented. Unlike in the case of eMI, the execution cue for the iMI is a hand picture presented on the screen to replace the cross. This picture was removed from the screen at time $\mathrm{t}=3000 \mathrm{~ms}$ independent of the response of the subject. This paradigm illustrating the sequence of events is shown on Fig. 1b. The subjects were asked to verbally express their laterality judgement of the presented hand pictures. They were instructed to say 'left' or 'right' if they judge the presented hand picture to be left or right lateralised respectively. The rational for using verbal response was to avoid hand movement which would occur if the subjects were told to push a button to supply their answers. The hand movement might interfere with experimental outcome [18]. The interference is not expected in the case of verbal response since the motor cortex area which controls the mouth movement is different from the hand area. The hand pictures had a size of 408 by 408 pixels and they show a hand performing various gestures on a plain background. For each gesture there was a left and a right hand picture. Each picture was presented in two possible orientations namely, counter-clockwise by $90^{\circ}(\mathrm{CCW})$ and clockwise by $90^{\circ}(\mathrm{CW})$. These produces four types iMI condition which are right $\mathrm{CCW}$ iMI, left $\mathrm{CW}$ iMI, right $\mathrm{CW}$ iMI and left CCW iMI. The first two types of iMI are termed medial orientations because they involve rotations towards the midline of the body. The last two are termed lateral orientations because they involve rotations outside the midline of the body [19]. Fig. 2 shows examples of the hand picture stimuli. This paradigm can be simulated online using our Google Chrome app at http://biomedsig.com/hlt/.

4) Procedure: Subjects were seated with a computer screen in front of them. They had their hands pronated and placed on a table in front of them and were instructed to relax. They were instructed to avoid physical movements during the tasks. The experimenter monitored the subjects throughout the experimental session making sure that instructions were followed. The experimenter requested for a part of the task to be repeated if instructions were not followed. The eMI trials took only about 20 minutes on average. Following the eMI trials and about 15 minutes of rest, the iMI trials followed. It was important to separate the eMI runs from the iMI runs. The reason for this was to reduce any interference of the techniques the subjects used between eMI and iMI in HLT [20]. A total of 120 trials for eMI were acquired 
a)
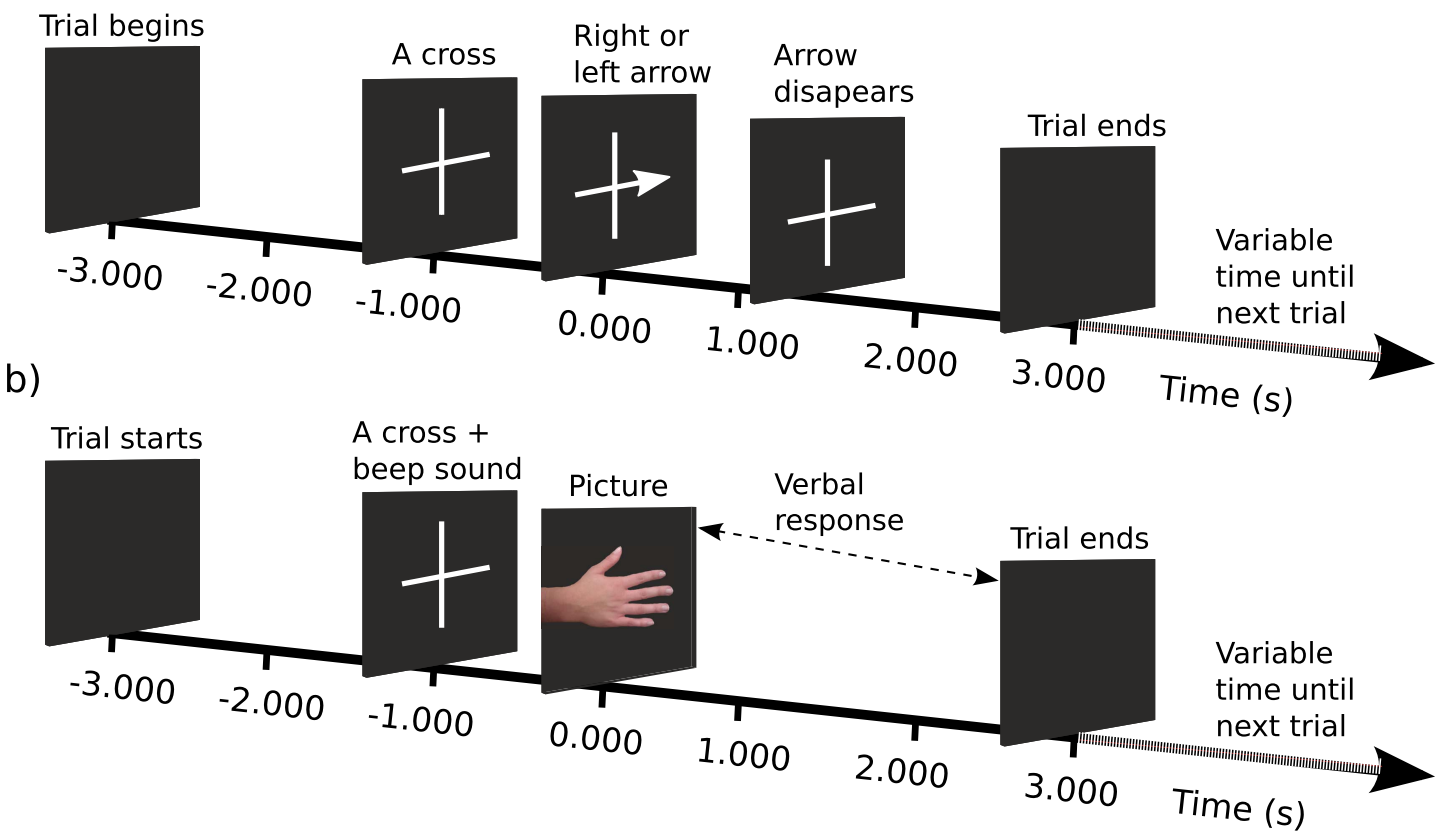

Fig. 1. The order of events for a) eMI and b) iMI trials or HLT trials.

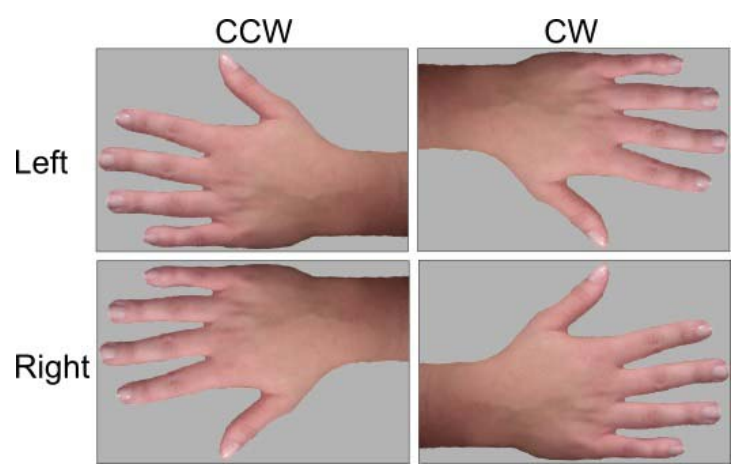

Fig. 2. The first row shows the left stimuli while the second row shows the right stimuli. The first column shows the images rotated counterclockwise (CCW) by $90^{\circ}$ while the second column shows images rotated clockwise $(\mathrm{CW})$ by $90^{\circ}$. The rotations are relative to the hands at upright position.

(comprising 60 trials for each of left and right hand). This was partitioned into four runs of 30 trials (comprising 15 trials for each hand shown in a random order). A total of 240 trials for iMI during HLT were acquired (comprising 60 trials for each of right $\mathrm{CCW}$, left $\mathrm{CW}$, right $\mathrm{CW}$ and left $\mathrm{CCW}$ ). This was partitioned into six runs of 40 trials (comprising 10 trials for each of the orientations shown in a random order). The subjects were instructed to rest between the runs. The number of trials was chosen to avoid fatigue.

5) Data Recording: Recording of data was carried out under MATLAB and Simulink (MATLAB R2012a, The MathWorks Inc., Natick, MA). Forty seven channels of EEG placed according to the international 10/10 electrode positioning standard as shown in Fig. 3 were used. Linked ear reference was used. The ground electrode was on location AFz. Electrooculogram (EOG) was recorded from an electrode placed on the lateral canthus, on the orbicularis oculi of the right eye. The EOG was recorded for the purpose of artefact

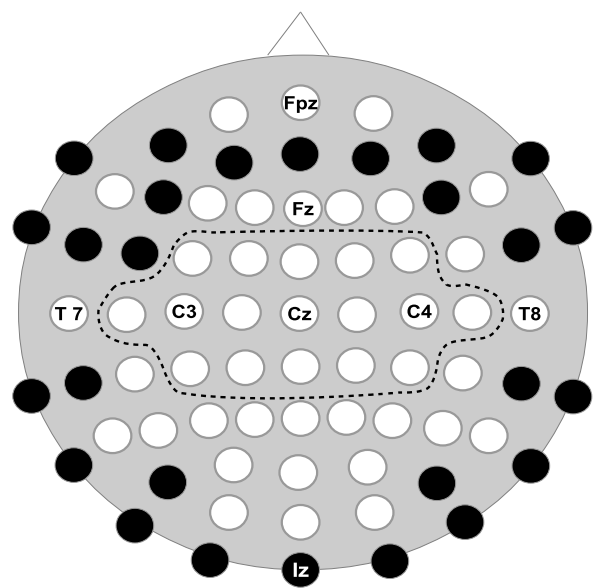

Fig. 3. The 10/10 international electrode positioning standard used for the experiment. The locations marked in black are unused. The electrodes inside the dashed shape are a subset of electrodes for an additional analysis.

detection. The EEG and EOG were acquired at a sample rate of $256 \mathrm{~Hz}$ with three modules of g.USBamp (biosignal amplifier, g.tec Medical Engineering $\mathrm{GmbH}$, Austria). Electrode impedance was kept below $5 \mathrm{k} \Omega$. The signal was filtered online with passband set between 0.5 and $60 \mathrm{~Hz}$ and a notch filter at $50 \mathrm{~Hz}$ using the filters built into the g.USBamp. In addition to EEG and EOG, audio signal was recorded during the iMI trials to allow the subjects to give their laterality judgement by verbal responses [21].

\section{B. Data Analysis}

The behavioural data, the responses and the response time of the subjects during the HLT (extracted by referencing the recorded audio signal) have already been analysed in a separate work. For the iMI, only the trials whose corresponding hand 
pictures were correctly identified by the subjects during HLT are used in the following analysis because we cannot be certain that mental rotation was used in the incorrectly identified trials. The median of correctly identified HLT trials are 97, 97, 93, and $93 \%$ of the left $\mathrm{CW}$, right $\mathrm{CCW}$, left $\mathrm{CCW}$ and right $\mathrm{CW}$ respectively [5].

1) Data Preprocessing: The recorded EEG data were visually inspected and bad epochs were removed. The logistic infomax independent component analysis (ICA) implemented in EEGLAB [22] was used to remove artefacts from the EEG by rejecting affected components. The artefacts included ocular, electrocardiographic and electromyographic artefacts which were identified by their typical morphology, spectrum and temporal characteristics. The remaining artefact free EEG data were bandpass filtered between 8 to $30 \mathrm{~Hz}$ using 97-taps Hamming-window based linear-phase finite impulse response filter. The average referencing of the electrodes was performed (by subtracting the average of all EEG electrodes from each EEG electrode) and the data for each trial (from -3000 to $3000 \mathrm{~ms}$ ) were split into segments of 100 samples. The resultant data were used in further analysis.

\section{Time-Frequency Analysis}

EEGLAB was used to compute, visualize and compare event related spectral perturbation (ERSP) [23] arising from eMI and iMI trials. The ERSP analysis was performed using the Morlet Wavelet transform in the frequency band 3-60 Hz. The Hanning-tapered window was applied and the number of cycles of the wavelet was set to 3 . These wavelet settings enabled low frequencies beginning from $3 \mathrm{~Hz}$ to be processed in a one second window [24]. The ERSP was calculated as power changes in decibels with reference to a baseline period $(\mathrm{t}=-2000$ to $-1000 \mathrm{~ms})$. The result is presented for a single channel and for all channels over the scalp.

1) Feature Extraction: The method of common spatial patterns (CSP) [25] was used to design spatial filters applied on pairs of classes to be classified. This CSP filtering minimises the variance of one of the classes while maximising that of the other. For the analysis of the CSP, two sets of electrodes were used. The first set, CSP1 consists of 47 electrode (all recording electrodes minus the EOG) and the second set, CSP2 consists of 17 electrodes around the sensorimotor areas which are those electrodes inside the dashed shape in Fig. 3.

The CSP filters were computed using data segments that fall within $\mathrm{t}=500-2500 \mathrm{~ms}$ following the execution cue or picture presentation. Data in these segments should provide the most significant discriminant features for classifying between classes.

The common spatial filters were used to filter all the data segments to obtain a new time series. The variance of this new time series was computed for each of the defined time segment of 100 samples (over the whole $6 \mathrm{~s}$ trial). The variance in each time segment formed features for classification purposes. In CSP method, the dimension of the features depends on the number of CSP filters ${ }^{1}$ used. Since it was difficult to decide

\footnotetext{
${ }^{1}$ Note that CSP filters are used in pairs; so $m$ number of CSP filters stands for $m$ first CSP filters and $m$ last CSP filters $(=2 m)$, with the CSP filter matrix sorted in the descending magnitude of the corresponding eigenvalues.
}

on a particular number of CSP filters to be used to build the features, the number of CSP filters was made a variable which ranges from two to the maximum number of CSP filters possible for each of CSP1 and CSP2.

2) Classification: Classification and evaluation procedures were performed using BioSig project [17]. For classifying the variance of the segmented CSP filtered data, the linear discriminant analysis (LDA) [26] method was used. In order to find the best segment of the data from where to obtain the training set, each of the segments used to obtain the CSP filters was individually used to perform initial classification without cross-validation. The segment with the highest Cohen's kappa [27] was chosen. Note that this initial classification is simply just a training data segment selection procedure used in the BioSig project [17]. It should not be confused with the classification of trials that will follow. Cohen's kappa ranges between 0 (pure chance) and 1 (perfect classification or agreement) [27]. Then classification employing the leaveone-out cross-validation followed. This procedure is as follows. At each step of the leave-one-out procedure, the trials belonging to a current training group were identified and the segment in each trial corresponding to the one identified in the initial classification was extracted for each class of data. This extracted data were then used to compute a 2-class LDA classifier. This LDA classifier built with the data from the selected segment of the training set were used to classify all the data samples of the testing set as either one class or the other.

The pairs of classes of data classified are, for hand eMI, left versus right hand; and for the iMI, left $\mathrm{CW}$ versus right $\mathrm{CCW}$, left $\mathrm{CW}$ versus right $\mathrm{CW}$, left $\mathrm{CCW}$ versus right $\mathrm{CCW}$ and left $\mathrm{CCW}$ versus right $\mathrm{CW}$. In addition to these, further pairs of classes for the iMI was classified. These are classification between the left $\mathrm{CCW}$ vs left $\mathrm{CW}$ and the right $\mathrm{CCW}$ versus right $\mathrm{CW}$. These two pairs of classification was performed in order to find if it is possible to decode the direction (medial versus lateral orientation) of the movement of the same hand in iMI.

The classification was assessed by computing Cohen's kappa and accuracy (the percentage of correctly classified trials) [27]. For each number of CSP filters, the confusion matrix [27] was computed at each classified data point along the trial. The confusion matrix was then used to compute the accuracy and the Cohen's kappa as functions of time and number of CSP filters. The segment along the trial with the best/maximum classification accuracy was obtained as the one with the highest Cohen's kappa allowing kappa to validate the accuracy. The value of the maximum classification accuracy and the time of the corresponding segment are presented as functions of number of CSP filters.

3) Statistics: For each CSP filter, paired sample Wilcoxon signed rank test was performed to compare the maximum classification accuracy between eMI and iMI. To correct for the multiple comparison due to testing at each CSP filter (recall that the number of CSP filter is a variable), the test significance level was treated with the Holm-Bonferroni correction [28]. Furthermore, a one-way ANOVA was conducted on averages across CSP filters of the maximum classification accuracy. The time corresponding to the maximum classification accu- 


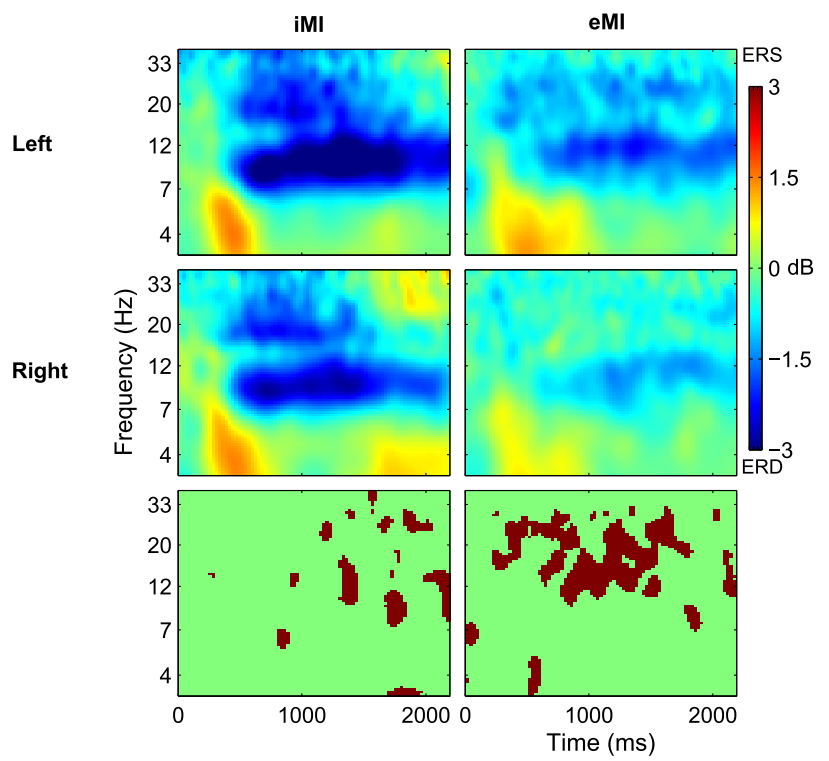

Fig. 4. ERSP result from channel location $\mathrm{C} 4$ for left and right eMI and, left $\mathrm{CCW}$ and right $\mathrm{CW}$ iMl. The last row shows statistically significant difference (shaded area) between left and right for each of eMI and iMl. The $\mathrm{x}$-axis shows the time along a trial where cue/picture was presented at $\mathrm{t}=0$. Holm's correction for multiple comparison was applied. The data were averaged across all subjects $(n=15)$.

racy was averaged across CSP filters and compared between eMI and iMI using paired t-test. In all the statistical tests, $\mathrm{n}=15$ and the uncorrected statistical significance level was set to 0.05 .

\section{Results}

\section{A. Time-Frequency Analysis}

Example ERSP result from channel location $\mathrm{C} 4$ is presented in Fig. 4. For iMI, the figure shows results for left CCW (Left) and right CW (Right). The last row of Fig. 4 shows statistically significant difference (shaded area) between left and right for each of eMI and iMI. The $\mathrm{x}$-axis shows the time along a trial where cue/picture was presented at $\mathrm{t}=0$.

From Fig. 4, ERSP desynchronisation (ERD) showing cortical activity began at about $500 \mathrm{~ms}$ following cue presentation. The ERSP has frequencies between $7-12 \mathrm{~Hz}$ and $16-30 \mathrm{~Hz}$. The low frequency ERSP synchronisation (ERS) at about $400 \mathrm{~ms}$ is likely due to the visual processing of the cue and picture.

Fig. 5 shows the scalp ERSP example for left and right hand eMI and, left CW and right CCW iMI. This figure was averaged across all subjects between $t=1000-1400 \mathrm{~ms}$ within $8-30 \mathrm{~Hz}$ frequency band. The last row of Fig. 5 highlights electrodes showing statistically significant left-right difference for each of eMI and iMI.

In both eMI and iMI, ERSP desynchronisation are present in the motor areas showing cortical activities in these areas during these imaginations. The ERSP activities are lateralised which is more clearly seen for the right hand where activities is lateralised towards the left hemisphere. This lateralisation of activities should contribute to left-right differences. The last row of Fig. 5 shows that as well as in eMI, left-right difference

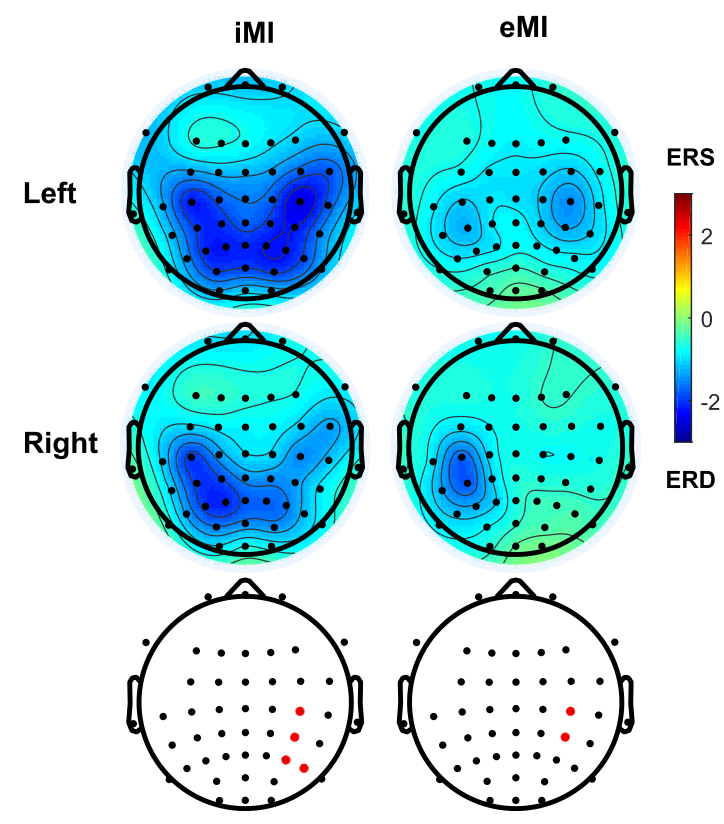

Fig. 5. Scalp ERSP example for left and right hand eMI and, left CW and right CCW iMl. This result was obtained between $t=1000-1400 \mathrm{~ms}$ within $8-30 \mathrm{~Hz}$ frequency band. The last row highlights electrodes showing statistically significant left-right difference for each of eMI and iMI. Correction for multiple comparison was performed using false discovery rate. The data were averaged across all subjects $(n=15)$.

exists for iMI. This difference is shown by the highlighted electrodes in the last row of the figure. The aim here is to use a classifier to quantify the left-right differences shown in the last row of Fig. 4 and Fig. 5.

\section{B. Classification}

The value of kappa and accuracy as a function of time and number of CSP filters (for CSP1) is presented in the left column of Fig. 6a-e. In Fig. 6, a) is for left versus right MI; b), left $\mathrm{CW}$ versus right $\mathrm{CCW}$ iMI; c), left $\mathrm{CW}$ versus right $\mathrm{CW}$ iMI; d), left CCW versus right $\mathrm{CCW}$ iMI and e), left $\mathrm{CCW}$ versus right $\mathrm{CW}$ iMI.

Prior to the presentation of the execution cue in eMI and hand picture presentation in iMI during HLT $(t<0)$, the values of kappa were low indicating low performance or chance level separability of the classes. Although one of the reasons that kappa has low values in these regions was because data from the regions were not used in the training set. However, the data in this region were not expected to show class specific differences. In the case of eMI, the values of kappa indicate that the best performance occurs for 6-12 CSP filters at about $500 \mathrm{~ms}$ following the execution cue. In the case of the iMI, unlike in the eMI condition kappa reaches high values after about $1000 \mathrm{~ms}$ into task execution. These results support previous findings [5] where left-right differences in the eMI and iMI conditions start at about $500 \mathrm{~ms}$ and $1000 \mathrm{~ms}$ respectively following cue presentation. The number of CSP corresponding to the high kappa values for iMI is similar to that of eMI.

Since kappa was used to determine the maximum classification accuracy, the accuracy should have high values at the 
a)

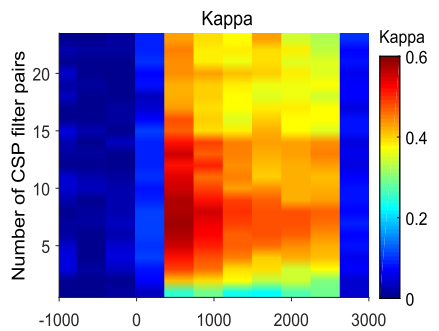

b)
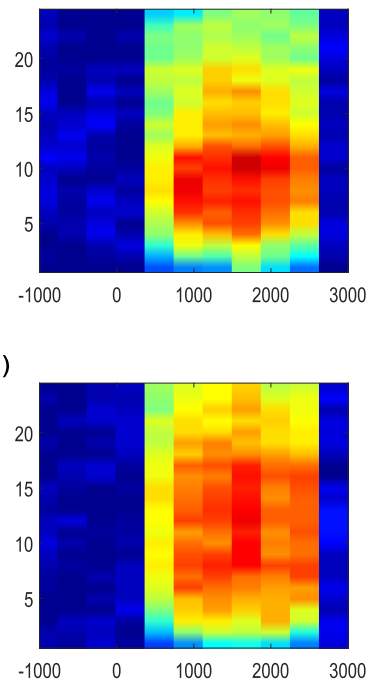

d)

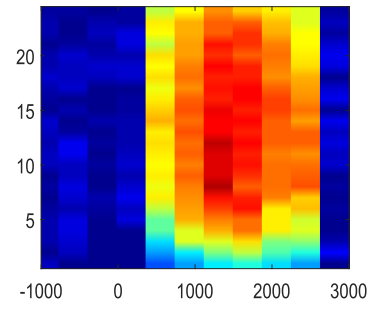

e)

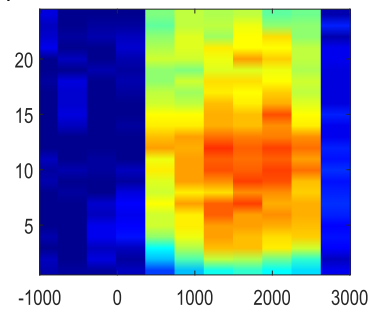

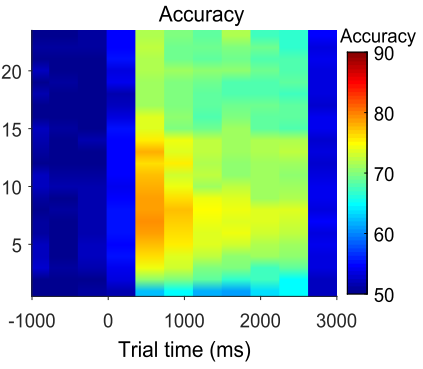
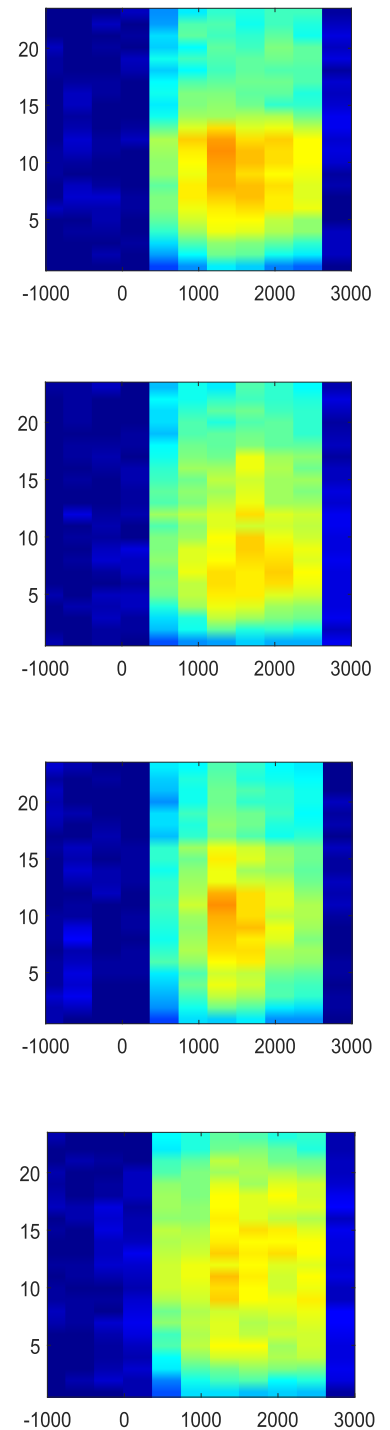

Fig. 6. The kappa (first column) and accuracy (second column) values for CSP1. The colour-coded legend on the right given the values of kappa and accuracy. Recall that the time $t=0 \mathrm{~ms}$ is the time when the execution cue was presented. The data were averaged across all subjects $(n=15)$ Each small rectangle unit in the figure has a height of 2 CSPs and a width of 100 samples. a) eMI left vs right. b) iMI left CW vs right CCW. c) iMl left CW vs right CW. d) iMI left CCW vs right CCW. e) iMI left CCW vs right $\mathrm{CW}$.

time when kappa has high values in order for kappa to be a good determinant. In order to show that this is the case the classification accuracies as a function of time into the trial and the number of CSP filters is presented in the right

column of Fig. 6. These figures have similar characteristics as the corresponding kappa image on the left of the figure indicating that accuracies were obtained at the right times. These characteristics have already been described for kappa.

The maximum classification accuracy as a function of the number of CSP filters is shown in Fig. 7 for both eMI and iMI. In order to easily compare the eMI with iMI, the accuracy for eMI is repeatedly plotted with each of the iMI combinations.

For both eMI and iMI, the maximum classification accuracy is low when the number of CSP filters used was below four. The accuracy peaked when the number of CSP filters was about between 6-12. After about 15 CSP filters classification accuracy decreased. The general relationship observed here where accuracy increases and then decreases with increasing number of CSP filters is reported in another study [29]. At each number of CSP filter, statistical comparison showed no significant difference between the maximum accuracy for the eMI and each of the iMI combinations.

In order to perform further analysis, the maximum classification accuracy was averaged between 6-12 CSP filters. All the classifications between condition pairs are high between these CSP filters. The average is shown in Table I. One-way ANOVA of the data in Table I needed a correction because the Mauchly's Test of Sphericity showed that the assumption of sphericity is violated, $\chi^{2}(9)=26.717, \mathrm{p}=0.002$. With a Greenhouse-Geisser correction the ANOVA revealed no main effect $(\mathrm{F}(2.3,32.195)=0.729, \mathrm{p}=0.508)$ suggesting no difference in classification accuracy between eMI and iMI or between the pairs (as shown in Table I) of iMI. These results further suggest that eMI is highly similar to iMI during HLT.

The time along the trial when the maximum difference appear between the classes (i.e. the time at which maximum classification was determined) was computed for both eMI and iMI. The times (relative to the start of the trials) which were averaged across 6-12 CSPs and across the subjects are, for left versus right eMI, $3.9 \pm 0.3 \mathrm{~s}$; left $\mathrm{CW}$ versus right $\mathrm{CCW}$, $4.6 \pm 0.4 \mathrm{~s}$; left $\mathrm{CW}$ versus right $\mathrm{CW}, 4.5 \pm 0.4 \mathrm{~s}$; left $\mathrm{CCW}$ versus right $\mathrm{CCW}, 4.5 \pm 0.4 \mathrm{~s}$; and with left $\mathrm{CCW}$ versus right $\mathrm{CW}, 4.6 \pm 0.5 \mathrm{~s}$. Statistical comparison performed on the average across CSP filters revealed that the difference in time is significant. The p-values showing that the time is greater for iMIs when compared with left versus right eMI are as follow: with 'left $\mathrm{CW}$ versus right $\mathrm{CCW}$ ', $\mathrm{p}=0.001$; 'left $\mathrm{CW}$ versus right $\mathrm{CW}$, $\mathrm{p}=0.002$; 'left $\mathrm{CCW}$ versus right $\mathrm{CCW}$,' $\mathrm{p}=0.003$; and with 'left $\mathrm{CCW}$ versus right $\mathrm{CW}$ ' $\mathrm{p}=0.001$. The reason for this difference is published [5] and it is simply because classification between conditions peaks for the iMI only after a decision on the presented hand was made. This is unlike in the case of eMI where no such a decision is required; imagination of movement begins soon after execution cue presentation since the correct hand is immediately identified for eMI.

The classification results for eMI and iMI are compared and presented once again but this time the electrodes used for the classification are restricted to those closest to the sensorimotor cortex areas (CSP2). Following this reduction in the number of channels the classification accuracy for the iMI dropped with respect to that of eMI but this was not 

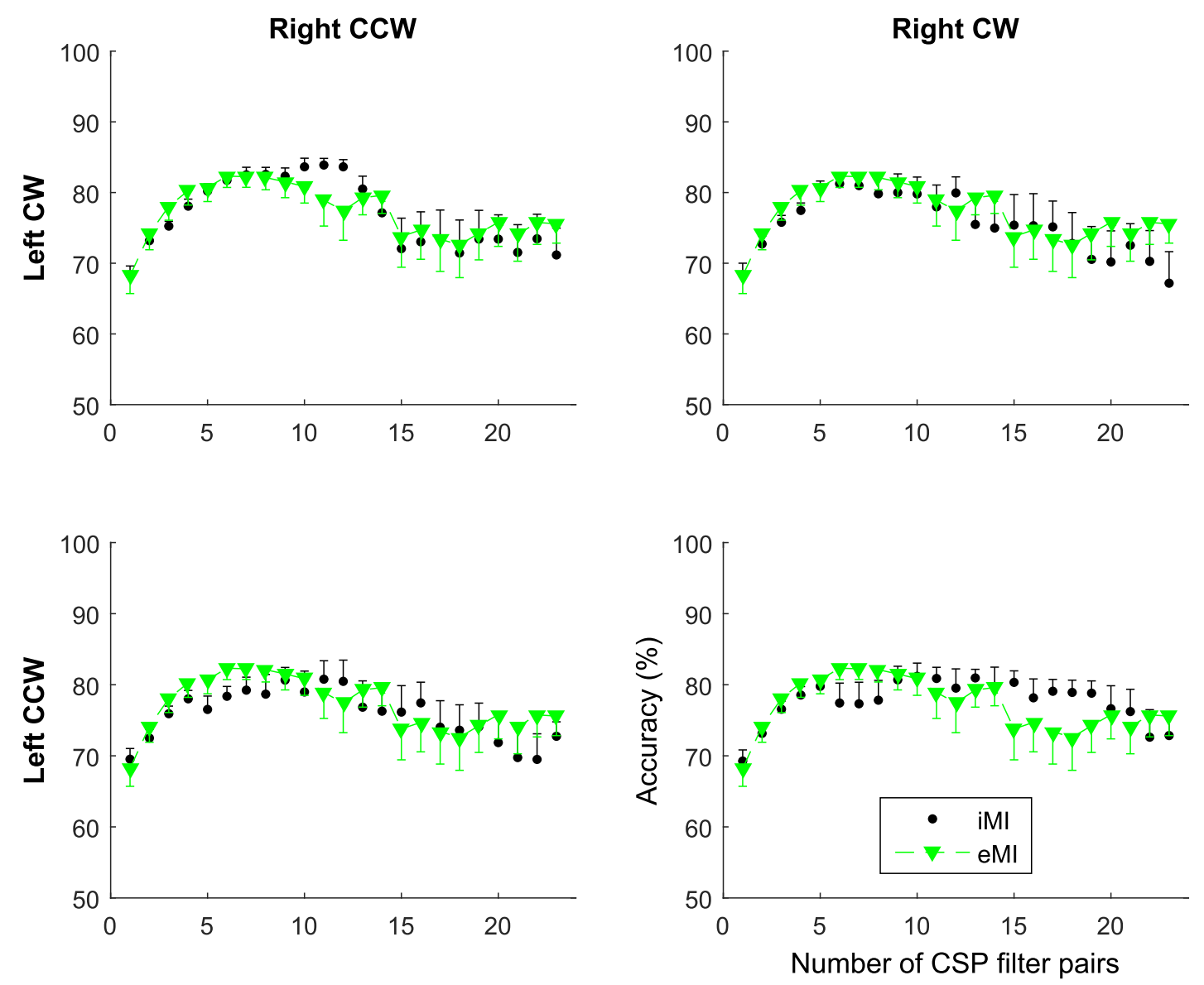

Fig. 7. Maximum classification accuracy values as a function of the number of CSPs (CSP1) for iMl and eMI. Since there is only one set of results for eMI, it is repeatedly plotted for each combination of iMl to ease visual comparison. To reduce cluttering, only one 'hand' of the error bars is shown. Each data point is an average across all subjects $(n=15)$.

TABLE I

The Maximum Classification Accuracy Averaged Between 6-12 CSP Filters (CSP1) for eMI and iMI

\begin{tabular}{|c|c|c|c|c|c|}
\hline \multirow[t]{2}{*}{ Subj.ID } & Explicit MI & \multicolumn{4}{|c|}{ Implicit MI } \\
\hline & $\begin{array}{l}\text { Right vs } \\
\text { Left }\end{array}$ & $\begin{array}{l}\text { Right CCW vs } \\
\text { Left CW }\end{array}$ & $\begin{array}{l}\text { Right CW vs Left } \\
\text { CW }\end{array}$ & $\begin{array}{l}\text { Right CCW vs Left } \\
\text { CCW }\end{array}$ & $\begin{array}{l}\text { Right CW vs Left } \\
\text { CCW }\end{array}$ \\
\hline 1 & 82 & 85 & 84 & 83 & 83 \\
\hline 2 & 84 & 87 & 81 & 84 & 79 \\
\hline 3 & 76 & 81 & 59 & 83 & 82 \\
\hline 4 & 80 & 81 & 82 & 83 & 78 \\
\hline 5 & 83 & 80 & 88 & 78 & 84 \\
\hline 6 & 85 & 80 & 80 & 80 & 77 \\
\hline 7 & 87 & 76 & 78 & 79 & 77 \\
\hline 8 & 70 & 87 & 82 & 49 & 50 \\
\hline 9 & 96 & 85 & 87 & 84 & 83 \\
\hline 10 & 79 & 84 & 87 & 83 & 84 \\
\hline 11 & 89 & 84 & 81 & 86 & 84 \\
\hline 12 & 81 & 86 & 81 & 84 & 79 \\
\hline 13 & 77 & 78 & 71 & 78 & 79 \\
\hline 14 & 79 & 83 & 78 & 77 & 81 \\
\hline 15 & 64 & 86 & 83 & 84 & 90 \\
\hline Avg (STD) & $81(8)$ & $83(3)$ & $80(7)$ & $80(9)$ & 79 (9) \\
\hline
\end{tabular}

statistically significant except for one CSP filter (number of CSPs $=2$ pairs for Left CW vs Right $\mathrm{CW}$ ) which does not correspond to the maximum classification accuracy. The classification results averaged across subjects are shown in Table II.
The maximum classification results are as follow. For the Left vs Right eMI, $76.0 \pm 2.1 \%$ (5 CSPs); Left CW vs Right CCW, $72.3 \pm 1.1 \%$ (7 CSPs); Left CW vs Right CW, $71.5 \pm 1.3 \%$ (3 CSPs); Left CCW vs Right CCW, 
TABLE II

Classification Accuracies Using CSP2 for Different Number of CSPs. Each Condition Was Averaged Across the 15 Subjects

\begin{tabular}{|c|c|c|c|c|c|c|c|c|}
\hline Conditions & $1 \mathrm{CSP}$ & $2 \mathrm{CSP}$ & $3 \mathrm{CSP}$ & $4 \mathrm{CSP}$ & $5 \mathrm{CSP}$ & $6 \mathrm{CSP}$ & $7 \mathrm{CSP}$ & $8 \mathrm{CSP}$ \\
\hline $\begin{array}{r}\text { Explicit MI } \\
\text { Left vs Right } \\
\text { Implicit MI }\end{array}$ & $73.0 \pm 2.4$ & $74.0 \pm 2.4$ & $74.8 \pm 2.3$ & $75.3 \pm 2.3$ & $76.0 \pm 2.1$ & $74.5 \pm 2.0$ & $75.3 \pm 2.0$ & $75.5 \pm 1.9$ \\
\hline Left CW vs Right CCW & $65.5 \pm 1.3$ & $69.8 \pm 1.1$ & $70.9 \pm 1.1$ & $71.5 \pm 1.4$ & $71.9 \pm 1.4$ & $71.4 \pm 1.3$ & $72.3 \pm 1.1$ & $70.4 \pm 0.9$ \\
\hline Left CW vs Right CW & $67.5 \pm 1.4$ & $70.6 \pm 1.1$ & $71.5 \pm 1.3$ & $71.4 \pm 1.0$ & $70.4 \pm 1.2$ & $71.2 \pm 1.3$ & $71.0 \pm 1.1$ & $70.9 \pm 0.9$ \\
\hline Left CCW vs Right CCW & $67.8 \pm 1.3$ & $68.8 \pm 1.3$ & $70.9 \pm 1.0$ & $70.4 \pm 0.9$ & $70.4 \pm 1.3$ & $71.3 \pm 0.9$ & $70.6 \pm 1.2$ & $70.8 \pm 1.1$ \\
\hline Left CCW vs Right CW & $68.6 \pm 1.2$ & $70.2 \pm 1.3$ & $71.6 \pm 1.1$ & $71.6 \pm 1.2$ & $72.0 \pm 1.4$ & $71.2 \pm 1.4$ & $71.3 \pm 1.5$ & $70.0 \pm 1.5$ \\
\hline
\end{tabular}

$71.3 \pm 0.9 \%$ (6 CSPs); and for the Left CCW vs Right CW, $72.0 \pm 1.4 \%$ (5 CSPs). An interesting result is that the standard errors corresponding to the iMI during HLT are smaller than that of eMI $(\mathrm{p} \ll 0.001)$. This is possibly because the implicit imagination in mental rotation is an unconscious process which tends to occur similarly in the subjects. This is unlike eMI which is a conscious process [30] and depends on a subject's ability to imagine [11].

Further classifications performed (using all 47 EEG channels) in order to see if there is a difference between $\mathrm{CW}$ and $\mathrm{CCW}$ movement of the same hand is presented (i.e. within hand classification). The trend shown by the accuracy over number of CSP filters by this within hand classification is similar to that shown in Fig. 7. The classification accuracy reached $81 \pm 7 \%$ (at $12 \mathrm{CSPs}$ ) for the left CCW versus left $\mathrm{CW}$ and $78 \pm 7 \%$ (at $8 \mathrm{CSPs}$ ) for the right $\mathrm{CCW}$ versus right $\mathrm{CW}$. This suggests that the direction of implicitly imagined movement can be recognised.

\section{DISCUSSIONS}

This paper demonstrates that comparable offline classification accuracy can be achieved in iMI and eMI. Furthermore, high classification accuracies of both forms of motor imagery were preserved when only electrodes over the central cortex was used indicating that both iMI and eMI activate sensorimotor cortex. The most unexpected result of the study is that lateral and medial mental rotation of hand produce distinctive spatial patterns that can be classified with an average accuracy higher than $80 \%$.

We have shown in this study the feasibility of a novel BCI neurorehabilitation paradigm based on iMI. Implicit motor imagery was achieved by asking subjects to judge the laterality of hand pictures in HLT. Previous studies have shown that unlike visual imagery which is not subject to biomechanical constrains [31], judging the laterality of the images of hands includes the embodiment process, i.e. it is performed from the egocentric perspective [32]. Thus, response time increases and ERD intensity increases while a person tries to judge laterality of a hand in an orientation that is biomechanically hard to perform [31].

In this study we used the same type of features and classification methods for both iMI and eMI, achieving similar performances. The main difference was that the time along a single trial at which maximum classification was reached occurred later for iMI. This is because in the case of eMI, a participant begins motor imagination of the chosen hand soon after the execution cue presentation since a hand is explicitly stated. However during iMI in HLT, the subject goes first through a decision process to identify the hand. The decision process takes time and may involve individual comparison of each hand with the presented picture of a hand. Thus the EEG recording immediately following picture presentation may contain traces of activation relating to both hands, leading to poor classifier performance. This is in accordance with our previous study based on time-frequency and current source localisation analysis of this dataset [5].

The design of our experiment matched requirements of BCI used for rehabilitation purposes. We presented participants with only two most natural orientations, medial and lateral, to facilitate iMI, and maximise ERD. We generated the four used orientations of the hands (right $\mathrm{CW}$, right $\mathrm{CCW}$, left $\mathrm{CW}$ and left $\mathrm{CCW}$ ) from the same image; therefore a person could not memorise and associate a hand with the orientation. To make the task more engaging we included images of hands in different gestures. We used simple gestures which do not involve an object. However, the set of images used for the 'graded motor imagery' programme, which is commercially available, may contain more complex gestures which are introduced at certain stages during the course of the programme.

A person with motor deficit needs to be able to operate a rehabilitative BCI. To allow the possibility of a person with motor deficit to take part in HLT and also to avoid the influence of any other motor action of the upper limbs, we asked participants to provide verbal responses instead of pressing buttons as it is often done in this type of study [18]. This method of verbal response provided us with a unique opportunity of acquiring non-confounded EEG data which would otherwise be confounded with a real motor activity of the hands.

Although reaction time was shorter for the medial (right $\mathrm{CCW}$ and left $\mathrm{CW}$ ) than for the lateral (right $\mathrm{CW}$ and left $\mathrm{CCW}$ ) orientation, there was no statistically significant difference in left-right classification accuracy for any of four possible combinations. In all cases, highest accuracy was achieved in a time widow $1200-1400 \mathrm{~ms}$ post cue. Another explanation is that for the lateral orientations, some subjects are not able to produce significant differences in EEG to classify the orientations due to the difficulty involved in identifying the hands in these orientations. Although the difference in reaction time did not have a significant effect on the average classification accuracy, it may have affected some of 
the classification accuracies of some subjects e.g. subject 3 (Right CW vs Left CW) and 8 (Right CCW vs Left CCW and Right $\mathrm{CW}$ vs Left $\mathrm{CCW}$ ). These subjects may not be able to produce significant differences in EEG to classify the lateral orientations due to the difficulty involved in identifying the hands in these orientations which comprise movement outside of the body centre.

Previous studies [5], [7] including a study from our group [5], reported strong activation of the parietal cortex, involved in general spatial rotation of objects. In order to confirm involvement of the sensorimotor cortex, which is a prerequisite for a rehabilitation strategy aiming at restoration of movement, we performed classification of both eMI and iMI based on 17 electrodes located across the FC, C and CP sagittal lines. Although classification accuracy was slightly lower for this case, there was no statistically significant difference in classification accuracies between eMI and iMI, for a range of classifiers based on different number of CSPs.

An unexpected result of the study was a relatively high classification accuracy between medial and lateral orientation of a single hand. This can add to the complexity of the neurorehabilitation training task and maintain patient's interest in training. A previous study based on independent component derived from EEG showed that it is possible to differentiate between imagined flexion and extension of a single hand [33]. The CSP used in this study are similar to independent components in a sense that both can create an irregular spatial pattern over the skull that is most representative for the imagined movement.

In this study, classification was performed offline; in an online version, BCI should provide a visual feedback to the participant to indicate the right answer. Unlike in eMI, an instant feedback with a bar pointing to the left or to the right might be distracting as iMI does not involve conscious motor action. Still, evidence from studies on healthy people indicate that even without feedback, prolonged practice of HLT improves performances in hand laterality judgement [34]. In case of patients, a feedback would be required as a form of encouragement and guidance. Furthermore, HLT which induces iMI is game-like which allows it to support motivation in a movement rehabilitation setting.

This BCI paradigm based on iMI through mental rotation could also be used for training of stroke patients with a spatial neglect. A study [35] showed that patients with unilateral neglect are capable of directing attention to the neglected side in order to perform mental rotation. Images of hand could therefore be presented in the visual filed of the non-neglected side while iMI could promote practicing mental rotation of the affected side. While for the rehabilitation of movement one would ideally create BCI classifier based on the activity of the sensorimotor cortex, for improving spatial awareness a BCI classifier should classify the activity of the parietal cortex [36].

Future work includes investigation into techniques, filtering methods and choice of number of electrodes and their locations that can improve classification accuracy in mental rotation. Following this would be an online mental rotation based BCI built for rehabilitation purposes.

\section{CONCLUSIONS}

This study has shown that similar left-right classification accuracy can be achieved in eMI and iMI. The study also showed that it was possible to detect the direction of implicitly imagined movement. These are significant results which go further to support the involvement of MI in mental rotation. The results also suggest that iMI in mental rotation may be employed in rehabilitation of movement and bodily spatial awareness, thus having comparable and potentially even larger application in neurorehabilitation than eMI.

\section{REFERENCES}

[1] L. F. Nicolas-Alonso and J. Gomez-Gil, "Brain computer interfaces, a review," Sensors, vol. 12, no. 2, pp. 1211-1279, 2012. [Online]. Available: http://dx.doi.org/10.3390/s120201211

[2] A. A. Frolov, D. Húsek, E. V. Biryukova, P. D. Bobrov, O. A. Mokienko, and A. V. Alexandrov, "Principles of motor recovery in post-stroke patients using hand exoskeleton controlled by the brain-computer interface based on motor imagery," Neural Netw. World, vol. 27, no. 1, pp. 107-137, 2017.

[3] C. Guger, G. Edlinger, W. Harkam, I. Niedermayer, and G. Pfurtscheller, "How many people are able to operate an EEG-based brain-computer interface (BCI)?" IEEE Trans. Neural Syst. Rehabil. Eng., vol. 11, no. 2, pp. 145-147, Jun. 2003.

[4] K. K. Ang et al., "Clinical study of neurorehabilitation in stroke using eeg-based motor imagery brain-computer interface with robotic feedback," in Proc. Annu. Int. Conf. IEEE Eng. Med. Biol. Soc. (EMBC), Aug. 2010, pp. 5549-5552.

[5] B. A. Osuagwu and A. Vuckovic, "Similarities between explicit and implicit motor imagery in mental rotation of hands: An EEG study," Neuropsychologia, vol. 65, pp. 197-210, Dec. 2014. [Online]. Available: http://dx.doi.org/10.1016/j.neuropsychologia.2014.10.029

[6] A. D. Walz, T. Usichenko, G. L. Moseley, and M. Lotze, "Graded motor imagery and the impact on pain processing in a case of CRPS," Clin. J. Pain, vol. 29, no. 3, pp. 276-279, 2013. [Online]. Available: http://journals.lww.com/clinicalpain/Fulltext/2013/03000/Graded Motor_Imagery_and_the_Impact_on_Pain.10.aspx

[7] J. M. Zacks, "Neuroimaging studies of mental rotation: A metaanalysis and review," J. Cognit. Neurosci., vol. 20, no. 1, pp. 1-19, 2008.

[8] L. M. Parsons, "Temporal and kinematic properties of motor behavior reflected in mentally simulated action," J. Experim. Psychol., Human Perception Perform., vol. 20, no. 4, pp. 709-730, 1994.

[9] C. Neuper, R. Scherer, M. Reiner, and G. Pfurtscheller, "Imagery of motor actions: Differential effects of kinesthetic and visual-motor mode of imagery in single-trial EEG," Cognit. Brain Res., vol. 25, no. 3, pp. 668-677, Dec. 2005.

[10] F. Di Pietro, T. R. Stanton, G. L. Moseley, M. Lotze, and J. H. McAuley, "Interhemispheric somatosensory differences in chronic pain reflect abnormality of the healthy side," Human Brain Mapping, vol. 36, no. 2, pp. 508-518, 2015. [Online]. Available: http://dx.doi.org/10.1002/hbm. 22643

[11] A. Vuckovic and B. A. Osuagwu, "Using a motor imagery questionnaire to estimate the performance of a brain-computer interface based on object oriented motor imagery," Clin. Neurophysiol., vol. 124, no. 8, pp. 1586-1595, 2013. [Online]. Available: http://dx.doi.org/10.1016/j. clinph.2013.02.016

[12] M. Gregg, C. Hall, and A. Butler, "The MIQ-RS: A suitable option for examining movement imagery ability," Evidence-Based Complementary Alternative Med., vol. 7, no. 2, pp. 249-257, 2010.

[13] C. Dettmers, M. Benz, J. Liepert, and B. Rockstroh, "Motor imagery in stroke patients, or plegic patients with spinal cord or peripheral diseases," Acta Neurol. Scandinavica, vol. 126, no. 4, pp. 238-247, 2012.

[14] L. M. Parsons, "Imagined spatial transformations of one's hands and feet," Cognit. Psychol., vol. 19, no. 2, pp. 178-241, 1987.

[15] G. L. Moseley, D. S. Butler, T. B. Beames, and T. J. Giles, The Graded Motor Imagery Handbook. Adelaide, SA, Australia: Noigroup Publications, 2012

[16] A. K. Thompson and J. R. Wolpaw, "Operant conditioning of spinal reflexes: From basic science to clinical therapy," Frontiers Integrat. Neurosci., vol. 8, p. 25, Mar. 2014. 
[17] A. Schlögl and C. Brunner, "BioSig: A free and open source software library for BCI research," Computer, vol. 41, no. 10, pp. 44-50, Oct. 2008.

[18] K. Takeda, N. Shimoda, Y. Sato, M. Ogano, and H. Kato, "Reaction time differences between left- and right-handers during mental rotation of hand pictures," Laterality, vol. 15, no. 4, pp. 415-425, 2010. [Online]. Available: http://dx.doi.org/10.1080/13576500902938105

[19] L. M. Parsons, "Imagined spatial transformation of one's body," J. Experim. Psychol., General, vol. 116, no. 2, pp. 172-191, 1987.

[20] M. Wraga, W. L. Thompson, N. M. Alpert, and S. M. Kosslyn, "Implicit transfer of motor strategies in mental rotation," Brain Cognit., vol. 52, no. 2, pp. 135-143, 2003.

[21] S. Ionta, A. D. Fourkas, M. Fiorio, and S. M. Aglioti, "The influence of hands posture on mental rotation of hands and feet," Experim. Brain Res., vol. 183, no. 1, pp. 1-7, 2007. [Online]. Available: http://dx.doi.org/10.1007/s00221-007-1020-2

[22] A. Delorme and S. Makeig, "EEGLAB: An open source toolbox for analysis of single-trial EEG dynamics including independent component analysis," J. Neurosci. Methods, vol. 134, no. 1, pp. 9-21, Mar. 2004. [Online]. Available: http://www.sciencedirect.com/ science/article/pii/S0165027003003479

[23] S. Makeig, "Auditory event-related dynamics of the EEG spectrum and effects of exposure to tones," Electroencephalogr. Clin. Neurophysiol., vol. 86, no. 4, pp. 283-293, 1993.

[24] R. K. Young, Wavelet Theory and Its Applications, vol. 189. Cambridge, MA, USA: Kluwer, 1993.

[25] H. Ramoser, J. Müller-Gerking, and G. Pfurtscheller, "Optimal spatial filtering of single trial EEG during imagined hand movement," IEEE Trans. Neural Syst. Rehabil. Eng., vol. 8, no. 4, pp. 441-446, Dec. 2000.

[26] K. Fukunaga, Introduction to Statistical Pattern Recognition, 2nd ed. London, U.K.: Academic, 1990.
[27] M. Billinger et al., "Is it significant? Guidelines for reporting BCI performance," in Towards Practical Brain-Computer Interfaces, B. Z. Allison, S. Dunne, R. Leeb, J. D. R. Millán, and A. Nijholt, Eds. Berlin, Germany: Springer-Verlag, 2012, pp. 333-354. [Online]. Available: https://link.springer.com/chapter/10.1007/978-3-642-29746-5_17

[28] S. Holm, "A simple sequentially rejective multiple test procedure," Scandin. J. Statist., vol. 6, no. 2, pp. 65-70, 1979.

[29] A. Vuckovic et al., "The influence of central neuropathic pain in paraplegic patients on performance of a motor imagery based Brain Computer Interface," Clin. Neurophysiol., vol. 126, no. 11, pp. 2170-2180, 2015.

[30] M. Jeannerod, "Neural simulation of action: A unifying mechanism for motor cognition," Neuroimage, vol. 14, no. 1, pp. S103-S109, 2001. [Online]. Available: http://dx.doi.org/10.1006/nimg.2001.0832

[31] R. Shepard and J. Metzler, "Mental rotation of three-dimensional objects," Science, vol. 171, no. 3972, pp. 701-703, 1971.

[32] L. A. Cooper and R. N. Shepard, "Mental transformation in the identification of left and right hands," J. Experim. Psychol., Human Perception Perform., vol. 1, no. 1, pp. 48-56, 1975.

[33] A. Vuckovic and F. Sepulveda, "Delta band contribution in cue based single trial classification of real and imaginary wrist movements," Med. Biol. Eng. Comput., vol. 46, no. 6, pp. 529-539, 2008.

[34] D. H. Uttal et al., "The malleability of spatial skills: A meta-analysis of training studies," Psychol. Bull., vol. 139, no. 2, pp. 352-402, 2013.

[35] S. D. Sala, R. H. Logie, N. Beschin, and M. Denis, "Preserved visuospatial transformations in representational neglect," Neuropsychologia, vol. 42, no. 10, pp. 1358-1364, 2004.

[36] T. Hassa, M. A. Schoenfeld, C. Dettmers, C. M. Stoppel, C. Weiller, and R. Lange, "Neural correlates of somatosensory processing in patients with neglect," Restorative Neurol. Neurosci., vol. 29, no. 4, pp. 253-263, 2011 